\title{
Comparación de oxímetros para detección de cardiopatías congénitas críticas
}

\author{
Comparison of oximeters for the detection of critical congenital heart diseases
}

\author{
Rocío A. Peña-Juarez ${ }^{1 *}$, Jorge A. Chávez-Saenz², Adrián García-Canales², Miguel A. Medina-Andrade, \\ María T. Martínez-González², Lorenzo Gutiérrez-Cobiánn ${ }^{1}$, Daniela A. Mendoza-Silva², \\ Cesar A. Valerio-Carballo y Antonio F. Gallardo-Meza² \\ ${ }^{1}$ Departamento de Cardiología pediátrica; ${ }^{2}$ Departamento de Pediatría, Hospital General de Occidente. Zapopan, Jalisco, México
}

\begin{abstract}
Resumen
Introducción y objetivos: En algunos centros el tamiz cardíaco no se realiza con la justificación de no tener el oxímetro adecuado. Comparamos la efectividad de dos marcas de oxímetros para realizarlo. Métodos: En los neonatos a término del Servicio de alojamiento conjunto del Hospital General de Occidente en Zapopan Jalisco México de mayo a noviembre del 2018 se realizó tamiz cardíaco según las guías de la Academia Americana de Pediatría con dos oxímetros, el Masimo SET ${ }^{\odot}$ (aprobado por la FDA) y el ChoiceMMed ${ }^{\oplus}$, comparando su efectividad para la detección de cardiopatías congénitas críticas, el tiempo de toma y los falsos positivos. Resultados: En cada grupo se analizaron 1,022 pacientes; con el oxímetro Massimo SET ${ }^{\oplus}$ se obtuvieron 83 pruebas positivas (8.12\%) de las cuales 22 casos presentaron alguna cardiopatía (26.5\%), lo que representa una sensibilidad del $100 \%$, una especificidad del $93.9 \%$, un valor predictivo positivo del $26.5 \%$ y un valor predictivo negativo del 100\% (Odd Ratio [OR]: 0.73; IC 95\%: 0.6-0.8). Con el oxímetro ChoiceMMed ${ }^{\circledast}$ se obtuvieron 168 pruebas positivas (16.4\%), de las cuales 22 casos presentaron alguna cardiopatía (13.09\%), con una sensibilidad del 100\%, una especificidad del $85.4 \%$, un valor predictivo positivo del $13.09 \%$ y un valor predictivo negativo del $100 \%$ (OR: 0.86; IC $95 \%$ : 0.8-0.92). En cuanto al tiempo para realizar el tamiz cardíaco, la media en minutos del oxímetro Masimo SET ${ }^{\oplus}$ fue 5.38 y del oxímetro ChoiceMMed ${ }^{\circledast}$ fue 9.7 minutos. Conclusiones: El oxímetro ChoiceMMed ${ }^{\circledR}$ presentó mayor cantidad de falsos positivos y mayor tiempo de realización del tamiz cardíaco en comparación al Masimo SET ${ }^{\oplus}$, sin embargo, ambos presentan un valor predictivo negativo del $100 \%$, siendo igualmente útiles como método de detección de cardiopatías críticas.
\end{abstract}

Palabras clave: Oximetría de pulso. Cardiopatía congénita. Enfermedad crítica.

\begin{abstract}
Introduction and objectives: In some centers the pulse oximetry is not performed with the justification of lack of the adequate oximeter. We compared the effectiveness of two brands of oximeters to perform it. Methods: In neonates a term of the joint housing service of a Hospital General de Occidente in Zapopan Jalisco Mexico from May-November 2018, an
\end{abstract}

\section{Correspondencia:}

*Rocío Alejandra Peña-Juárez

Departamento de Cardiología Pediátrica del

Hospital General de Occidente

Av. Zoquipan 1050, Col. Seattle

Fecha de recepción: 02-01-2019

Fecha de aceptación: 24-02-2019

DOI: $10.24875 /$ ACM. 19000019
Disponible en internet: 08-05-2019 Arch Cardiol Mex. 2019;89(2):172-180 www.archivoscardiologia.com

E-mail: alepejz@gmail.com

1405-9940 @ 2019 Instituto Nacional de Cardiología Ignacio Chávez. Publicado por Permanyer México SA de CV. Este es un artículo Open Access bajo la licencia CC BY-NC-ND (http://creativecommons.org/licenses/by-nc-nd/4.0/). 
examination of the characteristics of the American Academy of Pediatrics with both oximeters (ChoiceMMed ${ }^{\circledR}$ and Masimo $S E T^{\oplus}$ ) was carried out, comparing the detection of critical congenital heart disease (CCC), time of intake and false positives. Results: In each group 1,022 patients were analyzed; with the Masimo SET ${ }^{\oplus}$ oximeter 83 positive tests were obtained (8.12\%), of which 22 cases had some heart disease (26.5\%), which represents a sensitivity of $100 \%$, specificity of $93.9 \%$, positive predictive value of $26.5 \%$ and negative predictive value of $100 \%$ (OR: $0.73 ; 95 \% \mathrm{Cl}: 0.6-0.8$ ). With the ChoiceMMed ${ }^{\oplus}$ oximeter, 168 positive tests were obtained (16.4\%), of which 22 cases had some heart disease (13.09\%), with a sensitivity of $100 \%$, specificity of $85.4 \%$, positive predictive value of $13.09 \%$ and negative predictive value $100 \%$ (OR: 0.86; $95 \%$ Cl: $0.8-0.92$ ). Regarding the time to perform the cardiac sieve, the mean in minutes of the Masimo SET ${ }^{\oplus}$ oximeter was 5.38 and the ChoiceMMed ${ }^{\varpi}$ oximeter was 9.7 minutes. Conclusions: The ChoiceMMed ${ }^{\oplus}$ oximeter contains a large number of false positives and a greater number of echocardiograms and comparatively longer cardiac screen printing with Masimo $\mathrm{SET}^{\oplus}$, however, both with a negative predictive value of $100 \%$ eliminating such excuses.

Key words: Pulse oximetry. congenital heart disease. Critical illness.

\section{Introducción}

Las cardiopatías congénitas críticas (CCC) son consideradas como un riesgo alto de colapso cardiovascular o incluso de muerte ${ }^{1}$. La prevalencia, epidemiología y el impacto tardío en el diagnóstico de cardiopatías críticas se han descrito en varias publicaciones ${ }^{2}$. Las CCC afectan aproximadamente a 2 de cada 1,000 nacimientos, por lo que se estima que nacen alrededor de 40,000 bebes con alguna CCC por año solo en EE.UU. y 1.35 millones alrededor del mundo. Estas cardiopatías representan alrededor del $40 \%$ de las muertes provocadas por malformaciones congénitas y la mayoría de las muertes por enfermedad cardiovascular que ocurren en el primer año de vida ${ }^{3}$. En México, entre 1990 y 2010 la mortalidad infantil ha disminuido del 24 al $11.8 \%$ por cada 1,000 nacimientos. Sin embargo, la mortalidad por cardiopatías congénitas se ha incrementado. En el año 2000, la mortalidad por cardiopatías congénitas fue de 2,596 niños menores de 1 año, representando el 6.7\% de todas las muertes en este grupo de edad. En el 2008 la mortalidad por cardiopatías congénitas aumentó a 2,848 niños; correspondiendo al $9.6 \%$ del total. Además, se sabe que existe un $30 \%$ de muertes por CCC que se atribuyen a errores en el diagnóstico o un diagnóstico tardío $0^{4}$. Un común denominador de las CCC es la presencia de hipoxemia manifestada como cianosis, no siendo notoria a simple vista en todos los recién nacidos (RN); por ello ha sido necesario realizar estudios no invasivos para la detección temprana de estos pacientes. En 2009, Granelli, et al. ${ }^{5}$, en un grupo de 39,821 RN introdujeron la oximetría de pulso para la detección de CCC con una sensibilidad adecuada. Posteriormente la Academia Americana de Pediatría en conjunto con la Asociación Americana del Corazón ${ }^{6}$ determinaron que algunos pacientes con cardiopatías críticas no se detectaban previo a su egreso hospitalario, resultando en una alta morbilidad y mortalidad. Por ello se recomendaba realizar sistemáticamente oximetría de pulso a RN asintomáticos después de las primeras 24 horas de vida, pero antes de su egreso hospitalario, para detectar cardiopatías críticas (Fig. 1). A partir de ese momento la oximetría de pulso se determinó como una herramienta útil no invasiva y de bajo costo para la detección de estos pacientes. En nuestro país se están realizando esfuerzos para la obligatoriedad de la realización del cribado de oximetría de pulso de CCC; sin embargo, aún no es obligatorio realizarlo. Uno de los principales obstáculos para llevarlo a cabo es el oxímetro de pulso con el que se realiza el cribado, ya que se sabe que existen múltiples factores que pudieran afectar la exactitud como el movimiento, baja perfusión, interferencia electromagnética, interferencia con la luz ambiental, variantes de la hemoglobina y la altura, entre otros ${ }^{7}$. Se deben considerar características puntuales del equipo de medición de oximetría de pulso para realizar el tamizaje de CCC, entre las cuales se incluye ${ }^{8}$ :

- Cumplir con la norma de la Organización Internacional de Normalización y ser aprobado por la Food and Drug Administration (FDA) para uso hospitalario en $\mathrm{RN}$.

- Adaptarse al movimiento del paciente y contar con un rendimiento adecuado en situación real.

- Utilizar sensores recomendados para su colocación en las extremidades neonatales y sin necesidad de medios de fijación que pudieran afectar la integridad de la piel. Los sensores para adultos no se deben utilizar en los RN.

- Garantizar el uso de sensores indicados para cada monitor.

Hasta el momento solo se dispone de dos dispositivos de medición de oximetría de pulso que cuentan con los requisitos recomendados por la FDA, que son el oxímetro Masimo SET ${ }^{\circledR}$ y el oxímetro Covien Nellcor ${ }^{\circledR 9}$; 


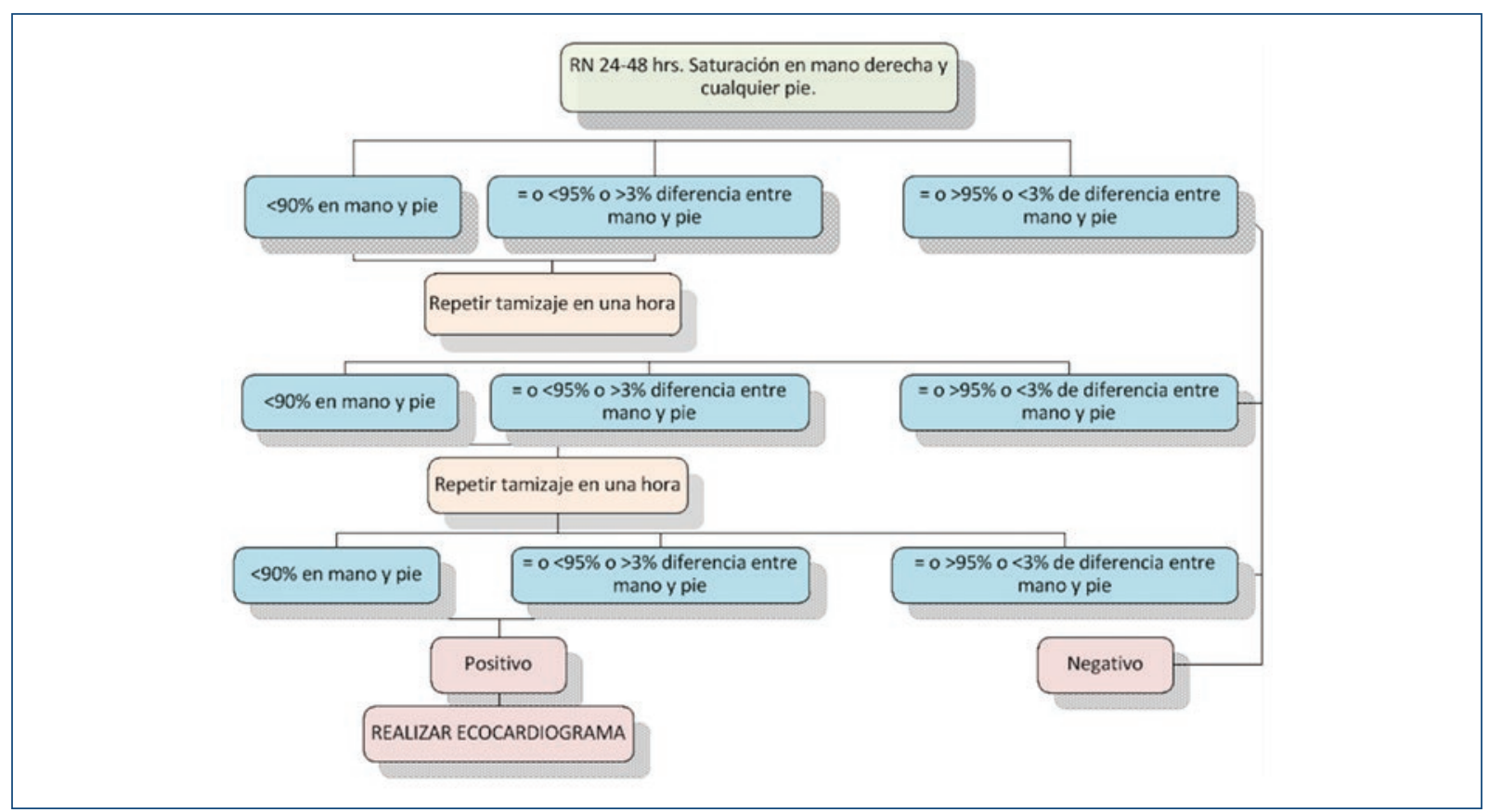

Figura 1. Algoritmo para tamiz cardiológico, Academia Americana de Pediatría (adaptada de Martin, et al., 2013). RN: recién nacido.

sin embargo, pocos centros en nuestro país cuentan con alguno de ellos, ya que su costo es elevado. Uno de los dispositivos más utilizados en el medio hospitalario es el oxímetro ChoiceMMed ${ }^{\circledR}$, que no está avalado por la FDA para el cribado de CCC pero es uno de los que más frecuentemente se utilizan para este fin. El objetivo de nuestro estudio es evaluar si el oxímetro ChoiceMMed $^{\circledR}$ tiene una eficacia similar como método de screening para CCC en comparación con uno de los oxímetros avalados por la FDA (Masimo SET ${ }^{\circledR}$ ).

\section{Métodos}

Se trata de un estudio prospectivo, longitudinal y observacional que se realizó de marzo a noviembre del 2018 en el Servicio de habitación conjunta en el Hospital General de Occidente en Guadalajara, Jalisco, México. Se incluyeron RN a término (37 a 41.6 semanas de gestación), con peso adecuado para la edad gestacional (mayor a 2,500 g) y mayores de 24 horas de vida, que se encontraban en el área de habitación conjunta. Fueron valorados por médicos pediatras y cardiólogos pediatras, los cuales les realizaban prueba de oximetría según el esquema de la Academia Americana de Pediatría. La prueba consiste en la medición de oximetría de pulso pre y posductal (extremidad superior derecha y cualquier extremidad inferior), posterior a las 24 horas de vida, en el cunero fisiológico, en RN de término aparentemente sanos. Un resultado se considera positivo si: a) cualquier medida de saturación es < 90\%; b) la saturación de oxígeno es < 95\% en ambas extremidades en 3 medidas separadas por 1 hora, 0 c) hay una diferencia absoluta $>3 \%$ en la saturación de oxígeno entre la mano derecha y el pie en las 3 mediciones, cada una separada por una hora. Cualquier medición que sea $>95 \%$ en cualquiera de las extremidades con una diferencia $<3 \%$ en la saturación de oxígeno entre las extremidades superior e inferior sería considerada un resultado negativo y el examen terminaría ${ }^{8,9}$ (Fig. 1). A todos los pacientes que ingresaron al estudio se les realizó tamizaje cardiológico con ambos oxímetros. Para ello se conformaron dos equipos médicos, que incluían un médico pediatra y un cardiólogo pediatra. Cada uno de los grupos realizaban las oximetrías con un equipo distinto; ya sea con el oxímetro Masimo $\mathrm{SET}^{\circledR}$ o con el oxímetro ChoiceM$\operatorname{Med}^{\circledR}$, con los cuales además de realizar prueba de oximetría se realizaba exploración física completa. En cuanto a la valoración ecocardiográfica, se realizaba al término de la valoración por ambos oxímetros; dichos estudios se realizaban con equipo Phillips Epic $7^{\circledR}$ con sonda 5 por un cardiólogo pediatra. Las variables para 


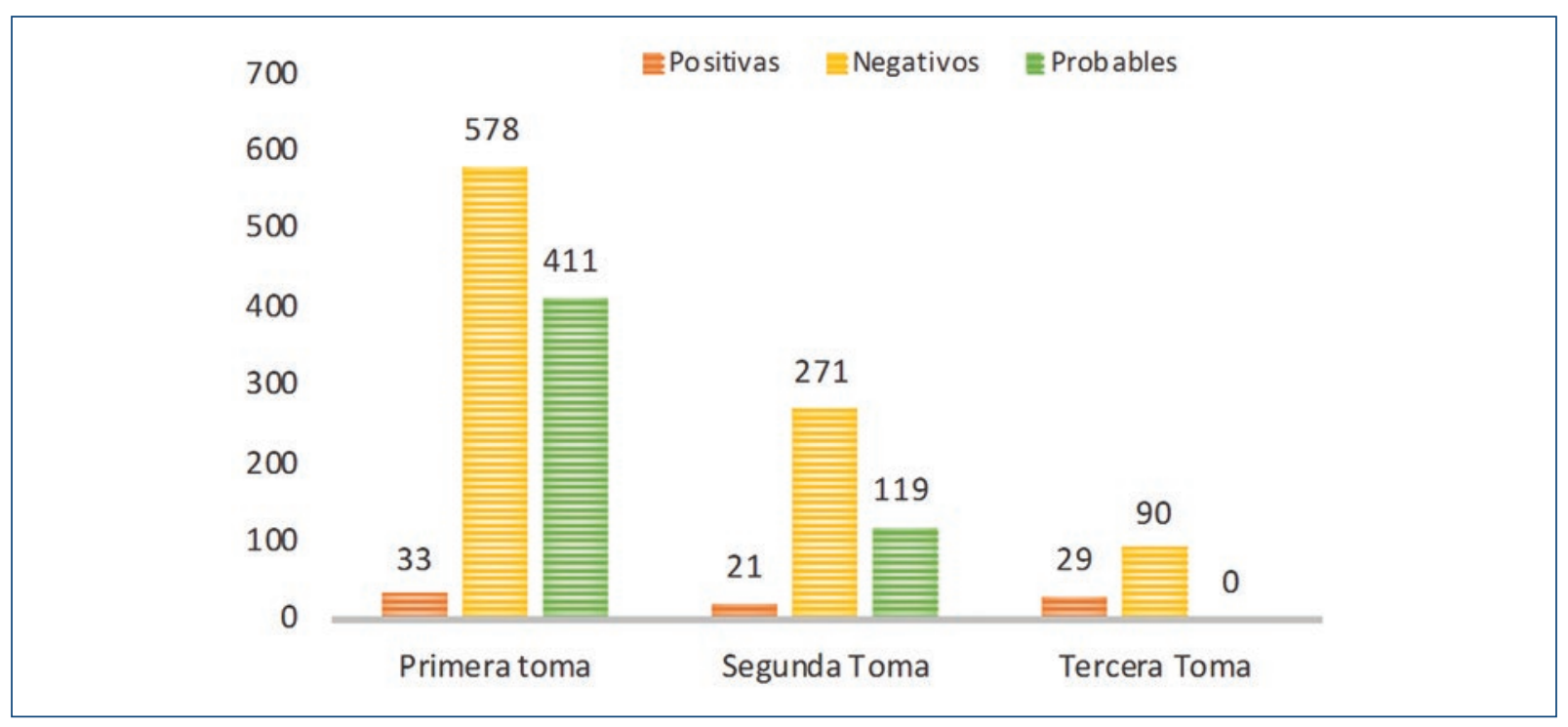

Figura 2. Resultados de tamiz cardiológico en las tres tomas con oxímetro Masimo $\mathrm{SET}^{\circledR}$.

estudiar fueron: el número de intentos (esto con base en el oxímetro Masimo SET ${ }^{\circledR}$, con el que se realiza la prueba en 3 minutos), las pruebas positivas, las pruebas falsas positivas y la realización de ecocardiograma en ambos grupos. Este estudio fue aprobado por el Comité de Investigación y Ética del Hospital.

\section{Resultados}

Durante el periodo de estudio, se evaluaron 1,022 pacientes. La edad de gestación promedio fue de $38.9 \pm$ 1.5 semanas y el peso promedio fue de $3,229 \mathrm{~g}(2,500 \mathrm{a}$ $4,500 \mathrm{~g})$. De los pacientes estudiados, 518 fueron varones (50.7\%). El 64.7\% $(n=661)$ nacieron por vía abdominal y en el $98.8 \%$ de los casos el embarazo fue único. Se valoró la presencia de patología materna, la cual se encontraba en el $2.3 \%$ de nuestra población, siendo la más frecuente la diabetes gestacional $(38 \%)$ seguida de la preeclampsia $(30 \%)$, el hipotiroidismo $(17 \%)$ y asma, lupus y síndrome de HELLP (4.1\% cada trastorno).

\section{Oxímetro Masimo SET ${ }^{\oplus}$}

\section{Primer registro}

En la primera toma de oximetría se ingresaron 1,022 pacientes, la saturación preductal promedio fue del $96.7 \%(81.9-100 \%)$, la saturación posductal promedio fue del $94.8 \%$ (78.3-99.8\%), con una diferencia de saturación promedio del $1.9 \%(6.5-20.8 \%)$. En cuanto a los índices de perfusión preductal el promedio fue de 2.5 (0.3-16) y posductal de $2.18 \%(0.1-18.8 \%)$. De las 1,022 oximetrías fueron positivas $33(3.2 \%)$, negativas $578(56.5 \%)$ y probables 411 (40.2\%) (Fig. 1). Los resultados ecocardiográficos fueron: normales 21 (63.6\%), hipertensión pulmonar $4(12.1 \%)$, cardiopatías no críticas $5(15.2 \%)$ y cardiopatías críticas $3(9.1 \%)$ ( 1 transposición de grandes arterias, 1 conexión anómala total de venas pulmonares y 1 coartación de la aorta). Se detectaron $3 \mathrm{RN}$ con datos de sepsis y 2 con datos de neumonía (Fig. 2).

\section{SEGUNDO REGISTRO}

Se ingresaron un total de 411 pacientes, de los cuales el $48.4 \%$ pertenecen al sexo femenino. La saturación preductal promedio fue del $96.38 \%(89-100 \%)$, mientras que el promedio de la posductal fue del $94.99 \%$ (81.6$99.8 \%$ ). La diferencia de saturación fue del 1.39\% (1.4$10.3 \%$ ), el índice de perfusión preductal fue del $2.44 \%$ (0.3-10.7\%) y el índice de perfusión posductal fue del $2.01 \%(0.2-10.1 \%)$. En cuanto a los resultados, 21 pruebas fueron positivas (5.1\%), 271 negativas $(65.9 \%)$ y 119 probables (29\%) (Fig. 1), y en los resultados de los ecocardiogramas encontramos un $24 \%(n=5)$ de cardiopatías no críticas y 16 normales; en este grupo se ingresaron 3 pacientes con datos de sepsis (Fig. 2).

\section{TERCER REGISTRO}

Se realizaron 119 pruebas de oximetría, de los cuales el $58 \%$ corresponden al sexo femenino. La saturación 


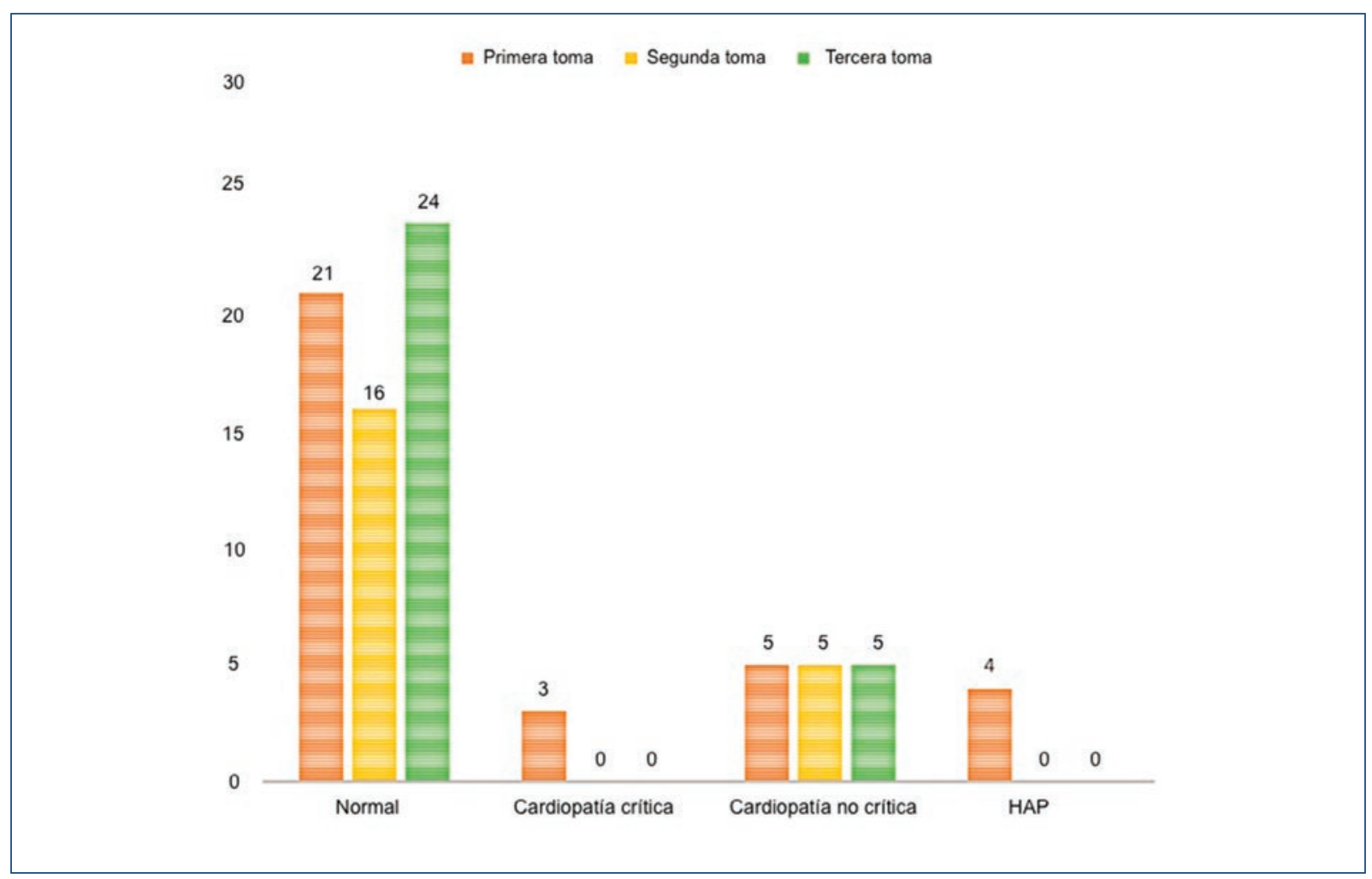

Figura 3. Resultados ecocardiográficos con oxímetro Masimo $\mathrm{SET}^{\circledR}$. HAP: hipertensión pulmonar.

preductal promedio fue del $96 \%(90.3-99.6 \%)$, la saturación posductal promedio fue del 94.4\% (89.7-98.7\%), la diferencia de saturación fue del 1.6\% (0.1-6.9\%). El índice de perfusión preductal fue del 2.5\% (0.6-9.6\%) y el índice de perfusión posductal del 2.1\% (0.5-9.6\%). En cuanto a los resultados, 29 pruebas resultaron positivas (24.3\%), de los cuales solo 5 pacientes (17.2\%) presentaron algún tipo de cardiopatía no crítica (Fig. 1). En el grupo de ecocardiogramas normales se detectaron 2 pacientes con datos de sepsis (Fig. 2).

En cuanto al número de intentos según el programa del oxímetro, que en promedio son 3 minutos, en el $60.1 \%$ (615 pacientes) fue solo un intento y el resto solo dos intentos; con una media de tiempo de 5.38 minutos.

\section{Oxímetro ChoiceMMed ${ }^{\circledR}$}

\section{Primer registro}

Se realizaron un total de 1,022 pruebas de oximetría, de las cuales la saturación preductal promedio fue del $96 \%(88-100 \%)$ y la saturación posductal fue del $93 \%$ (76-98\%). La diferencia de saturación es del 2.3\%
(1.8-16.4\%). En cuanto a los resultados, 37 pruebas resultaron positivas (3.6\%), negativas el $43.7 \%$ ( $n=$ 447) y el resto probable (Fig. 3). Los resultados del ecocardiograma fueron: normales $34(91.9 \%)$, hipertensión arterial pulmonar $1(2.7 \%)$, cardiopatía crítica 1 (2.7\%) (conexión anómala total de venas pulmonares) y cardiopatía no crítica 1 (2.7\%) (Fig. 4). Se detectaron 2 RN con datos de sepsis y 2 con neumonía.

\section{SEgundo REGISTRO}

Se realizaron 538 pruebas de oximetría, de las cuales 270 pacientes pertenecen al sexo femenino (50.18\%). La saturación preductal promedio fue del $95 \%$ (90-99\%), la saturación posductal fue del 93\% (81-99\%). En cuanto a la diferencia de saturación, el promedio fue del $2.42 \%$ (0-10.5\%). Del total de pruebas realizadas solo 30 resultaron positivas $(5.57 \%), 153$ negativas (28.4\%) y 355 probables (65.9\%) (Fig. 3). Los resultados del ecocardiograma fueron: cardiopatía crítica 1 (3.5\%) (transposición de grandes arterias), cardiopatía no crítica $1(3.5 \%)$ y el resto normales (93.3\%) (Fig. 4). Además, se detectaron 4 pacientes con datos de sepsis. 


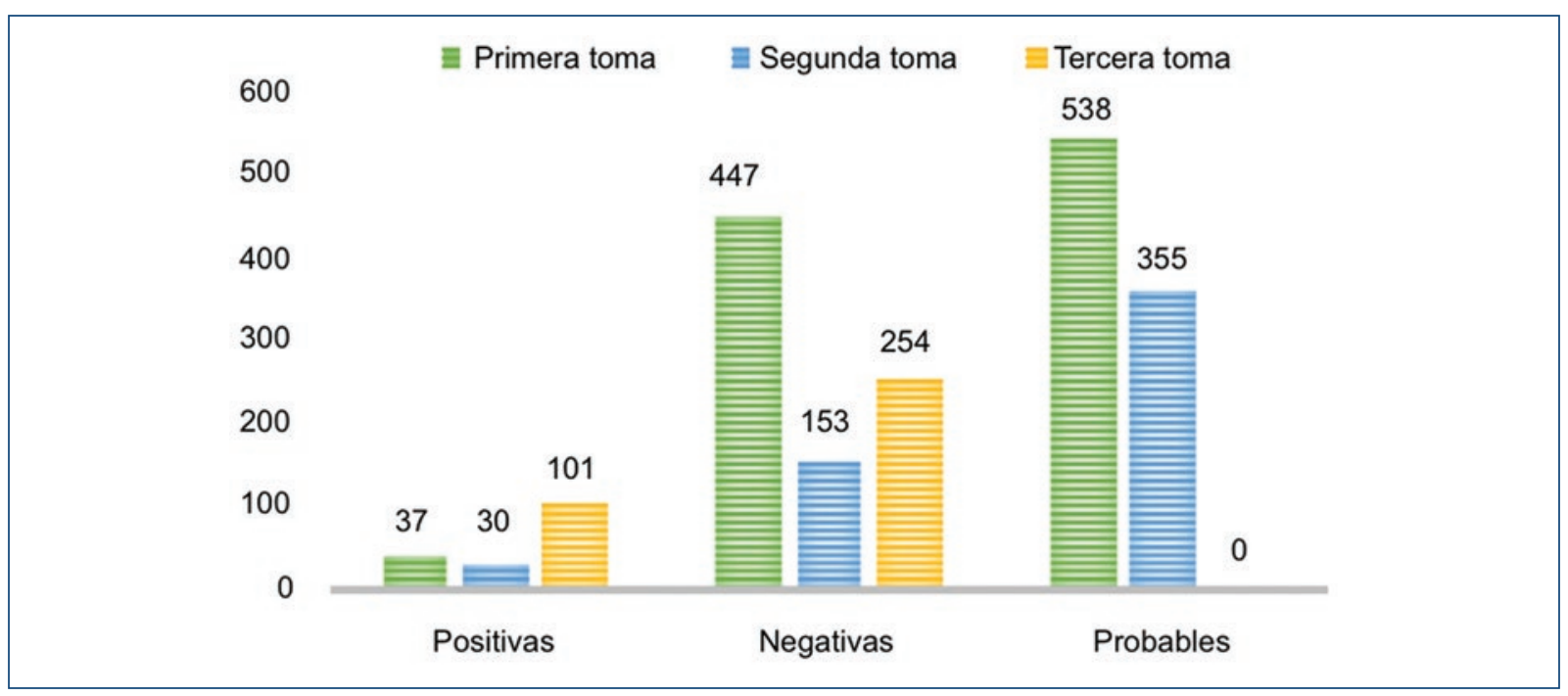

Figura 4. Resultados de pruebas de tamiz cardíaco con oxímetro ChoiceMMed ${ }^{\circledR}$.

\section{TERCER REGISTRO}

Se realizaron un total de 355 pruebas de oximetría, de las cuales el $50.4 \%(n=179)$ pertenecen al sexo femenino. La saturación preductal promedio fue del $94 \%(90-99 \%)$, la saturación posductal fue del $94 \%$ (86-97\%), con una diferencia de saturación del 1.5\% (0-7.8\%). En los resultados de la prueba de oximetría, 101 (28.4\%) fueron positivas (Fig. 3). En cuanto a los resultados ecocardiográficos fueron: con hipertensión arterial pulmonar $3(2.9 \%)$, cardiopatía crítica 1 (0.99\%) (coartación de la aorta), cardiopatía no críticas $13(12.87 \%$ ) y el resto normales (Fig. 4). Se detectaron 2 pacientes con datos de sepsis.

En cuanto al número de intentos, el $15 \%(n=153)$ requirieron 1 intento, el 37\% $(n=379) 2$ intentos, el $24.2 \%(n=248) 3$ intentos y el $23.8 \%(n=242)$ requirieron más de 4 intentos, con una media de tiempo de 9.7 minutos.

\section{Pruebas positivas}

Con el oxímetro Masimo SET ${ }^{\circledR}$, de las 1,022 pruebas de oximetría que se realizaron 83 fueron positivas $(8.12 \%)$, de las cuales 22 presentaron algún tipo de cardiopatía (26.5\%), lo que representa una sensibilidad del $100 \%$, una especificidad del $93.9 \%$, un valor predictivo positivo del $26.5 \%$ y un valor predictivo negativo del 100\% (OR: 0.73; IC 95\%: 0.6-0.8). En comparación con el oxímetro ChoiceMMed ${ }^{\circledR}$, de las 1,022 oximetrías realizadas, 168 fueron positivas (16.4\%), de las cuales
22 presentaron alguna alteración cardíaca (13.09\%), con una sensibilidad del $100 \%$, una especificidad del $85.4 \%$, un valor predictivo positivo del $13.09 \%$ y un valor predictivo negativo del 100\% (OR: 0.86; IC 95\%: 0.8-0.92) (Tabla 1).

\section{Discusión}

Desde septiembre del 2011 en los EE.UU. se indicó el screening para detección de $\mathrm{CCC}^{10}$; este es importante porque si estas patologías no se detectan a tiempo, se asocian a una mortalidad sumamente alta ${ }^{11}$. En México el tamiz cardiológico no está regulado de manera obligatoria; uno de los principales "obstáculos" que se reportan es el material y sobre todo el costo elevado de llevarlo a cabo. En cuanto a los costos del tamiz cardíaco, se han realizado varios estudios en los cuales se reporta que, en promedio, el costo por screening de CCC es de $\$ 250$ pesos; incluso en el estudio realizado en Nueva Jersey refieren un costo menor, de $\$ 92$ pesos, e incrementando un 3\% si requiere ecocardiograma y transporte según el estudio realizado en Minnesota ${ }^{9,12-14}$. En comparación, tratar un RN con una CCC puede suponer un mínimo de $\$ 895,000$ pesos $^{15}$, esto sin tomar en cuenta el alto porcentaje de mortalidad en este grupo de pacientes, como se ha mencionado previamente. Por lo tanto, el «costo elevado" de realizar el tamiz cardíaco no es una excusa para no realizarlo.

Otro punto importante que se refiere impide en algunas instituciones realizar el tamiz cardíaco es el 
Tabla 1. Comparación de eficacia para detección de cardiopatías congénitas críticas con ambos oxímetros

\begin{tabular}{|l|c|c|c|c|c|c|c|c|}
\hline Oxímetro & $\begin{array}{c}\text { Total de } \\
\text { tamizados }\end{array}$ & $\begin{array}{c}\text { Pruebas } \\
\text { positivas }\end{array}$ & $\begin{array}{c}\text { Ecos } \\
\text { anormales }\end{array}$ & Sensibilidad & Especificidad & $\begin{array}{c}\text { Valor } \\
\text { predictivo } \\
\text { positivo }\end{array}$ & $\begin{array}{c}\text { Valor } \\
\text { predictivo } \\
\text { negativo }\end{array}$ & OR IC 95\% \\
\hline Massimo SET $^{\circledR}$ & 1022 & 83 & 22 & $100 \%$ & $93.9 \%$ & 26.5 & 100 & $0.73(0.6-0.8)$ \\
\hline ChoiceMMed $^{\circledR}$ & 1022 & 168 & 22 & $100 \%$ & $85.4 \%$ & 13.09 & 100 & $086(0.8-0.92)$ \\
\hline
\end{tabular}

IC: intervalo de confianza; OR: Odd Ratio.

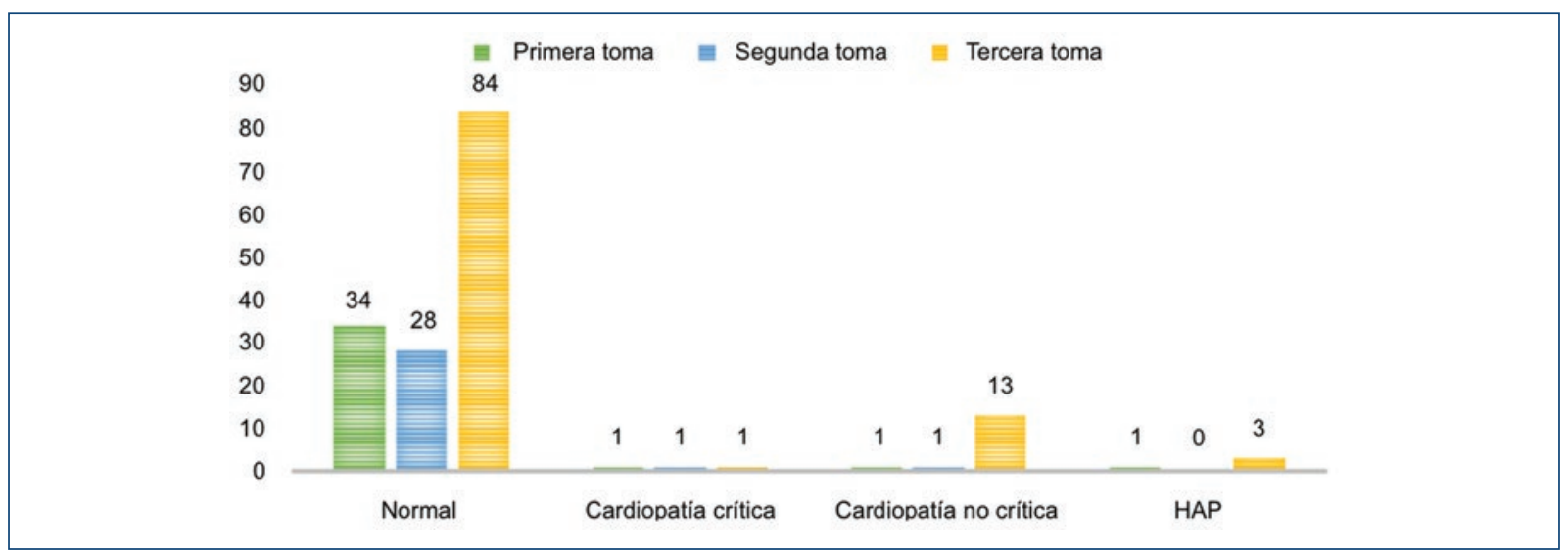

Figura 5. Resultados ecocardiográficos con oxímetro ChoiceMMed ${ }^{\circledR}$.

equipo. En nuestro estudio encontramos que ambos oxímetros presentan un valor predictivo negativo del $100 \%$; esto es muy similar a los estudios realizados por Riede, et al. ${ }^{16}$, donde se realizó oximetría de pulso en $41,445 \mathrm{RN}$ y se obtuvo un valor predictivo negativo del $99.99 \%$. Resultados similares se obtuvieron en la revisión sistemática de base de datos realizada por Plana Maria, et al. ${ }^{17}$, en la cual valoraron alrededor de 21 estudios donde se estudiaron 10,000 RN aparentemente sanos, con una sensibilidad del $76.3 \%$ y una especificidad del $99.9 \%$. En nuestro estudio encontramos resultados muy similares, si bien es cierto que el valor predictivo negativo de ambos oxímetros es del $100 \%$, lo que nos indica que ambos pueden detectar las CCC de una manera similar; sin embargo, con el equipo Massimo SET ${ }^{\circledR}$ el diagnóstico de las CCC se realizó en la primera toma, en comparación con el equipo ChoiceMMed ${ }^{\circledR}$ en el cual se diagnosticaron en la tercera toma.

En cuanto a los falsos positivos, en nuestro estudio observamos que en con el oxímetro Masimo SET ${ }^{\circledR}$ el valor predictivo positivo es del $26.5 \%$ y con el ChoiceMMed $^{\circledR}$ del $13.09 \%$, lo que indica que la cantidad de falsos positivos es moderada, por lo que se realizaron varios ecocardiogramas sin ser necesario, lo cual podría ocasionar más gastos, principalmente con el equipo ChoiceMMed ${ }^{\circledR}$. Por ello pudiera ser más rentable conseguir un equipo de Masimo $\mathrm{SET}^{\circledR}$, sin embargo en distintos estudios se ha observado que estos falsos positivos tienen mínimo impacto en la estimación de $\operatorname{costos}^{10}$ (Fig. 5).

En lo que corresponde al tiempo, encontramos que la media de tiempo con el equipo Masimo SET ${ }^{\circledR}$ fue de 5.38 minutos, y en la mayoría de los pacientes se requirió un solo intento para realizar la prueba, en comparación con el equipo ChoiceMMed ${ }^{\circledR}$, con el cual la media fue de 9.7 minutos, es decir casi el doble de tiempo, y en dos pacientes se requirió hasta 21 minutos para realizar una prueba de oximetría. El total de pruebas realizadas y sus resultados se muestran en la figura 6.

Otro punto importante que se tiene que analizar es la estimación del índice de perfusión con el equipo Masimo SET ${ }^{\circledR}$, el cual no es posible valorarlo con el equipo ChoiceMMed ${ }^{\circledR}$. El índice de perfusión es una evaluación de la fuerza pulsátil en un sitio específico de control y como tal es una medida indirecta y no invasiva de la perfusión periférica. El reconocimiento 


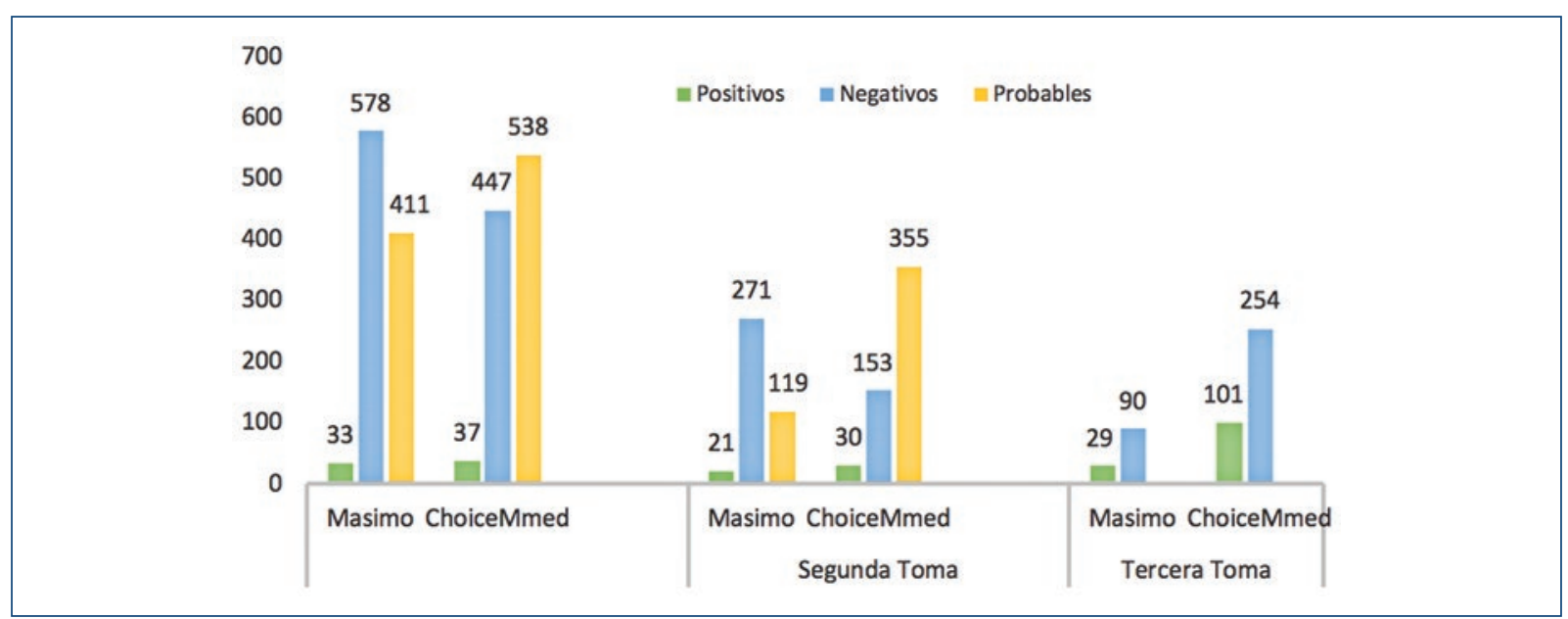

Figura 6. Total de pruebas realizadas con ambos oxímetros.

temprano del deterioro cardiocirculatorio es importante para evitar la hipoxia tisular, que en última instancia podría conducir a una insuficiencia multiorgánica. Hasta el momento los estudios clínicos que han evaluado la efectividad clínica del índice de perfusión han apoyado su aplicabilidad clínica, tal es el caso de su asociación con el diagnóstico de cardiopatías congénitas e incluso sepsis temprana ${ }^{18}$. En nuestro estudio con el índice de perfusión se detectaron de una forma más temprana cardiopatías no críticas, al igual que se detectaron pacientes con datos de sepsis.

\section{Conclusiones}

Encontramos que con ambos oxímetros la especificidad es alta, con una sensibilidad moderada para detectar CCC.

Sin embargo, creemos que se debe valorar la cantidad de tiempo para realizar la prueba (que es mayor con el equipo ChoiceMMed ${ }^{\circledR}$ ) y la cantidad de falsos positivos, lo cual puede llevar a incrementar los costos.

Además, como se mencionó previamente, si bien es cierto que en ambos oxímetros el valor predictivo negativo es similar, con el equipo Masimo $\mathrm{SET}^{\circledR}$ se detectaron las CCC de una forma más temprana; al igual que el índice de perfusión nos puede detectar de forma más temprana cardiopatías no críticas y datos de sepsis.

Existen múltiples estudios donde se ha determinado que el screening de oximetría de pulso para detectar cardiopatías críticas es adecuado, y obligado en algunos países. Se reducen los costos de atención y sobre todo la mortalidad en estos pacientes, por lo que creemos imperativo su realización, no siendo un impedimento el no contar con un oxímetro aprobado por la FDA.

\section{Financiamiento}

La presente investigación no ha recibido ayudas específicas provenientes de agencias del sector público, sector comercial o entidades sin ánimo de lucro.

\section{Conflicto de intereses}

Los autores declaran no tener conflicto de intereses.

\section{Responsabilidades éticas}

Protección de personas y animales. Los autores declaran que para esta investigación no se han realizado experimentos en seres humanos ni en animales.

Confidencialidad de los datos. Los autores declaran que han seguido los protocolos de su centro de trabajo sobre la publicación de datos de pacientes.

Derecho a la privacidad y consentimiento informado. Los autores declaran que en este artículo no aparecen datos de pacientes.

\section{Bibliografía}

1. Sailh AF, Hamawand AM, Sattar RA. Role of pulse oximetry screening for detection of life threatening congenital heart detects in newborns. Kurdistan Journal of Applied Research. 2018;3(2):134-9.

2. Bernier PL, Stefanescu A, Samoukovic G, Tchervenkov Cl. The challenge of congenital heart Disease worldwide. Epidemiologic and demographic facts. Semin Thorac Cardiovas Surg Pediatr Card Surg Annu. 2010;13:26-34.

3. Sola A, Golombek SG. Early detection with pulse oximetry oh hypoxemic neonatal conditions. Development of the IX Clinical Consensus State- 
ment of the Ibero-American Society of Neonatology (SIBEN). Int J Neonatal Screen. 2018;4:10

4. Torres-Cosme JL, Rolón-Porras C, Aguinaga-Ríos M, Acosta-Granado PM Reyes-Muñoz E, Murguía-Peniche T. Mortality from congenital heart disease in Mexico: A problema on the rise. PLoS One. 2016;11(3):e0150422.

5. de-Wahl Granelli A, Wennergren M, Sandberg K, Mellander M, Bejlum C, Inganäs $\mathrm{L}$, et al. Impact of pulse oximetry screening on the detection of duct dependent congenital heart Disease: a Swedish prospective screening study in 39821 newborns. BMJ. 2009;338:a3037.

6. Mahle WT, Newburger JW, Matherne GP, Smith FC, Hoke TR, Koppel R, et al.; American Heart Association Congenital Heart Defects Committee of the Council on Cardiovascular Disease in the Young, Council on Cardiovascular Nursing, and Interdisciplinary Council on Quality of Care and Outcomes Research; American Academy of Pediatrics Section on Cardiology and Cardiac Surgery, and Committee on Fetus and Newborn. Role of pulse oximetry in examining newborns for congenital heart disease: a scientific statement from the American Heart Association and American Academy of Pediatrics. Circulation. 2009; 120(5):447-58.

7. Mejia Salas H, Mejia Suarez M. Oximetría de Pulso. Rev Soc Bol Ped 2012:51(2):149-54.

8. Martin GR, Beekman RH $3^{\text {rd }}$, Mikula EB, Fasules J, Garg LF, Kemper AR, et al. Implementing recommenden screening for critical congenital heart disease. Pediatrics. 2013;132(1):e185-92.

9. Jiménez Carbajal MG, López-Perez D, Fernández Luna CP. Relevancia de la detección de cardiopatías congénitas complejas mediante cribado con oximetría de pulso en recién nacidos aparentemente sanos en los establecimientos de salud. Arch Cardiol Mex. 2018;88(4):298-305.

10. Peterson C, Grosse SD, Glidewell J, Garg LF, Van Naarden Braun K Knapp MM, et al. A public health economic assessment of hospitals'cost to screen newborns for critical congenital heart disease. Public Health Rep. 2014;129:86-93.

11. Carroll AE, Downs SM. Comprehensive cost-utility analysis of newborn screening strategies. Pediatric. 2006;117:S287-95.

12. Wong KK, Fournier A, Frutiman DS, Graves L, Human DG, Narvey M, et al. Canadian Cardiovascular Society/Canadian Pediatric Cardiology Association Position Statement on pulse oximetry screening in newborns to enhance detection of critical congenital heart Disease. Can J Cardiol. 2017;33:199-208.

13. Kochilas LK, Lohr JL, Bruhn, Borman-Shoap E, Gams BL, Pylipow M, et al. Implementation of critical congenita heart Disease in US newborns. Pediatic. 2013;132:e595-603.

14. Peterson C, Grosse SD, Glidewell J. A public health economic assessment of hospitals cost to screen newborns for critical congenital heart Disease. Public Health Rep. 2014;129:86-93.

15. Garson A, Allen H, Gersony W, Hohn AR, Pinsky WW, Mikhail O. et al. The cost of congenital heart disease in children and adults. A model multicenter assesssment of price and practice variation. Arch Pediatr Adolesc Med. 1994;148:1039-45.

16. Riede FT, Worner C, Dahnert I, Mockel A, Kostelka M, Schneider P. Efectiveness of neonatal pulse oximetry screening for detection of critical congenital heart disease in daily clinical, routine-result from a prospective multicenter study. Eur J Pediatr. 2010;169:975-81.

17. Plana MN, Zamora J, Suresh G, Fernandez-Pineda L, Thangaratinam S, Ewer AK. Pulse oximetry screening for critical congenital heart defects. Cochrane Database Syst Rev. 2018;3:CD011912.

18. De la Peña Sanabria, Ochoa Martelo M, Baquero Latorre H, Acosta-Reyes J. Indice de perfusión periférica en la $\mathrm{UCI}$ neonatal: una respuesta a la monitorización no invasiva del recién nacido crítico. Perinatol Reprod Hum. 2017;31(2):85-90. 